\title{
Randomization Can Be a Healer: Consensus with Dynamic Omission Failures
}

\author{
Henrique Moniz, Nuno Ferreira Neves, Miguel Correia, and Paulo Veríssimo \\ University of Lisboa, Faculdade de Ciências, LASIGE*
}

\begin{abstract}
Wireless ad-hoc networks are being increasingly used in diverse contexts, ranging from casual meetings to disaster recovery operations. A promising approach is to model these networks as distributed systems prone to dynamic communication failures. This captures transitory disconnections in communication due to phenomena like interference and collisions, and permits an efficient use of the wireless broadcasting medium. This model, however, is bound by the impossibility result of Santoro and Widmayer, which states that, even with strong synchrony assumptions, there is no deterministic solution to any non-trivial form of agreement if $n-1$ or more messages can be lost per communication round in a system with $n$ processes. In this paper we propose a novel way to circumvent this impossibility result by employing randomization. We present a consensus protocol that ensures safety in the presence of an unrestricted number of omission faults, and guarantees progress in rounds where such faults are bounded by $f \leq\left\lceil\frac{n}{2}\right\rceil(n-k)+k-2$, where $k$ is the number of processes required to decide, eventually assuring termination with probability 1.
\end{abstract}

\section{Introduction}

Wireless ad-hoc networks are being increasingly used in diverse contexts, ranging from casual meetings to disaster recovery operations. The ability of distributed processes to execute coordinated activities despite failures is important to distributed systems, including those based in wireless ad-hoc networks. Such coordination requires agreement among the processes, a problem that has taken many incarnations in the literature: consensus, Byzantine generals, and interactive consistency are just a few examples $[12,17,23]$. The prevalent aspect of these formulations is that at some point in their execution the processes involved have to agree on a common item of information.

In the traditional models for distributed systems, faults are static and component-bound, i.e., a fault is associated to a particular component that is forever considered faulty. The faulty component can be a process or a communication link (e.g., [23, 24]). These models are referred to as component failure models. For systems based on these models to operate correctly, a certain number of components must not exhibit failures during their entire operation time.

\footnotetext{
* This work was partially supported by the FCT through the Multiannual and the CMU-Portugal Programs.
} 
This approach, however, is not well adapted to wireless ad-hoc networks. First, in these environments, faults have a more dynamic and transient nature. The nodes are usually subject to momentary disconnection due to node mobility and other environmental phenomena such as electromagnetic interference, fading, collisions, etc. These events may result in message loss or corruption, but should not be sufficient to permanently assume a process or link as faulty, specially because they can possibly affect many processes during the lifetime of the system. Due to the emergence of wireless networks, there is an increasing need for models that accurately capture the reality of these environments.

Second, the openness of wireless ad-hoc networks provides a natural broadcasting medium, where the cost of transmitting a message to multiple processes can be just the same of transmitting it to a single process, as long as they are within communication range. To take advantage of this feature, it becomes necessary to depart from the common modeling assumption of reliable point-to-point channels, usually employed by the component failure models. Developing a system based on this assumption forces the implementation of end-to-end message delivery mechanisms (similar to TCP), which significantly increase the medium access contention, impairing the overall performance. The unreliability inherent to radio communications has to be dealt with in some other way. Models that assume unreliable communication links are more adjusted to wireless networking. Tolerance to message loss becomes integrated within the semantics of the algorithms, instead of being abstracted by typically inefficient implementations.

More adapted to the wireless ad-hoc environments is the communication failure model $[27,28]$. This model differs from the component failure models in the sense that it focuses on the effects of faults rather than their source. On message-passing systems, any failure, regardless of its nature, will ultimately manifest itself as transmission faults. For example, a process crash will manifest into a series of transmission omission faults with the crashed process as sender, and a process that is attacked and falls under the control of a maliciously adversary may manifest into a series of transmission corruption faults where the contents of the messages are modified relative to the original protocol. Such an approach implicitly allows every component of the system to eventually fail. The only restriction is placed on the number of faults that simultaneously manifest in the system.

Research in this model, however, has been limited mainly due to two fundamental reasons. When the model was introduced by Santoro and Widmayer in 1989, a stringent impossibility result came along with it [27]. This result applies to the $k$-agreement problem among $n$ processes, in which $k$ out of $n$ processes must agree on a binary value $v \in\{0,1\}$. The Santoro-Widmayer impossibility result applies to non-trivial agreement, i.e., for $k>\lceil n / 2\rceil$. It states that there is no finite time deterministic algorithm that allows $n$ processes to reach $k$-agreement if more than $n-2$ transmission failures occur in a communication step. This is a very discouraging result since the crashing of a single process necessarily results in $n-1$ transmission failures, rendering this form of agreement impossible. Moreover, this result is produced under strong time assumptions where both 
the processes' relative processing times and communication delays are bound by known constants (i.e., a synchronous system).

The second reason has probably to do with some lack of practical interest of this model prior to the emergence of wireless ad-hoc communication. For distributed systems based on wired networks, it was safe and convenient to assume end-to-end reliable delivery mechanisms, since the implementation of such mechanisms did not represent a significant performance overhead. Interestingly, these models are also bound by an impossibility result: the FLP result [13]. It states that consensus is impossible to solve deterministically in asynchronous systems (i.e., where there are no assumptions about the processes' relative processing times and communication delays) if just a single process can fail.

Thus, on one hand we have asynchronous systems, bound by the FLP impossibility result, where agreement is impossible even if communication is reliable. On the other hand, we have systems that are synchronous but the communication is unreliable so they are bound by the Santoro-Widmayer impossibility result, also making agreement impossible. While several solutions have been proposed over the years to circumvent the FLP result (e.g., partial synchrony models [10], failure detection [7], wormholes [21]), the result of Santoro and Widmayer, for the reasons stated above, has not received comparable attention. Nevertheless, getting past the current upper bound of $n-2$ transmission failures is paramount to the embracing of the communication failure model for emergent networking environments.

This paper proposes a protocol that circumvents the Santoro-Widmayer impossibility result in both a practical and efficient way. We achieve this by employing randomization, which has never been applied before in the context of the communication failure model. The Santoro-Widmayer impossibility result rules out deterministic solutions to agreement in this model. Randomization takes a probabilistic approach to the problem, and has been used in the past to solve consensus in FLP-bound systems (starting with $[3,25]$ ). It overcomes previous limitations by supplying processes with access to random information (e.g., a coin flip) and combining this with a refinement of the problem statement where a decision is ensured with a probability of 1 .

The paper describes a randomized binary $k$-consensus algorithm that tolerates omission faults. The algorithm allows at least $k$ processes to decide on a common binary value in a system with $n$ processes such that $k>\frac{n}{2}$. The safety properties of consensus (i.e., validity and agreement) are ensured even with an unrestricted number of faults, while the liveness property (i.e., termination) is ensured if the number of faults per round does not exceed $\left\lceil\frac{n}{2}\right\rceil(n-k)+k-2$. This algorithm is adequate for wireless ad-hoc networks because it allows one to take advantage of the broadcasting medium in an efficient way and, at the same time, ensures safety under severe communication problems that lead to many message losses. The termination is achieved with probability 1 when communication becomes stable, i.e., when the threshold above is satisfied. Furthermore, the algorithm is efficient in the sense that it is fast-learning [16], i.e., it terminates in 2 communication steps under favorable conditions (i.e., with no message 
losses, benign patterns of message losses, and/or all processes having the same initial value).

The remainder of the paper is organized as follows: Section 2 discusses the related work. Section 3 formalizes the $k$-consensus problem, and the next section presents the system model. Section 5 describes the algorithm, and the correctness proofs are provided in the following section. Section 7 discusses some performance aspects of the algorithm, and finally, Section 8 concludes the paper.

\section{Related Work}

The problem of reaching agreement with unreliable communication links goes back as far as 1975 when Akkoyunlu et al. pointed out that an agreement between two processes connected by unreliable communication paths leads to an infinite exchange of messages [2]. In 1978 Gray identified essentially the same problem by formulating the generals paradox [14]. He showed that there is no fixed length protocol that allows agreement between two processes connected through an unreliable communication link. This problem is often referred to as the coordinated attack problem from the formalization of Lynch [18]. Varghese and Lynch later proposed a randomized solution to the coordinated attack problem where the protocol runs for a fixed number of rounds and agreement is reached with a probability proportional to the number of rounds [30].

The previous result was generalized to an arbitrary number of processes by Santoro and Widmayer [27,28]. Their contribution provides an important impossibility result. It states that there is no fixed-time solution to the problem of $k$-agreement (i.e., $k>\left\lceil\frac{n}{2}\right\rceil$ processes decide the same value 0 or 1 ) in a system with $n$ processes if more than $n-2$ links are allowed to lose messages. Their problem statement represents a weaker form of agreement than ours. The definition of $k$-agreement allows processes to decide different values as long as $k$ decide the same value, while in our definition (i.e., $k$-consensus) no process is allowed to decide a different value.

The work of Chockler et al. presents algorithms that solve consensus in systems where nodes fail only by crashing and messages can be lost due to collisions [9]. Their solution assumes that processes have access to a collision detector that determines when message collisions occur, which allows nodes to take recovery measures when messages are lost. Message omissions other than those due to collisions, however, are not covered by their model. By contrast, our model assumes message omissions regardless of their nature.

Two other works also solve consensus under dynamic communication failures. The work of Biely et al. does so by addressing the problem in the context of the heard-of model of Charron-Bost and Schiper [4,8]. This model permits a finegrained specification of the fault patterns allowed in the system, thus being able to distinguish the cases where the fault pattern exceeds the lower bound of Santoro and Widmayer but is not harmful to the system as a whole (e.g., $n-1$ faults are harmful to the system if they originate at the same process, but may not be if they originate each one at a different process). The work of 
Schmid et al. presents an analogous contribution in the sense that it restricts the number of faults that each process may experience such that the harmful fault patterns are avoided [29]. None of these two contributions, however, deal with the problematic essence of the Santoro-Widmayer impossibility result, which is the failure of every transmission from a single process rendering consensus impossible. This implies that consensus remains unsolvable if, for instance, in a wireless ad-hoc network, a single node falls out of range of every other node for an unknown period of time.

Crash-recovery models based on failure detection mechanisms can also be applied to wireless environments because of their ability to capture the disconnection and eventual reconnection of processes $[11,15,22,1]$. The granularity of these models, however, was not intended to capture connectivity scenarios likely to arise in wireless environments. For example, consensus cannot be solved in scenarios where every good process (i.e., one that is not crashed) has some faulty link to another good process. Such configuration violates the eventual weak accuracy property required by failure detectors.

\section{The $k$-Consensus Problem}

The $k$-consensus problem considers a set of $n$ processes where each process $p_{i}$ proposes a binary value $v_{i} \in\{0,1\}$, and at least $k>\frac{n}{2}$ of them have to decide on a common value proposed by one of the processes. The remaining $n-k$ processes do not necessarily have to decide, but if they do, they are not allowed to decide on a different value. Our problem formulation is designed to accommodate a randomized solution and is formally defined by the properties:

Validity. If all processes propose the same value $v$, then any process that decides, decides $v$.

Agreement. No two processes decide differently.

Termination. At least $k$ processes eventually decide with probability 1 .

\section{System Model}

The system is composed by a fixed set of $n$ processes $\Pi=\left\{p_{0}, p_{1}, \ldots, p_{n-1}\right\}$. The timing model is assumed to be synchronous. This implies that (1) there is a known upper bound on time required by a process to execute a step, (2) there is a known upper bound on message transmission delays, and (3) every process has a local clock with a known bounded rate of drift with respect to real-time.

The communication between processes proceeds in synchronous rounds. At each round, every process $p_{i} \in \Pi$ executes the following actions: (1) transmits a message $m$ to every process $p_{j} \in \Pi$, including itself, by invoking broadcast $(m)$, (2) receives the messages broadcast in the current round by invoking receive (), and (3) performs a local computation based on its current state and the set of messages received so far. We should note that the assumption of a broadcast operation generating $n$ transmissions arises from the necessity of modeling the 
possibility of non-uniform message delivery by the processes. In practice, this operation can still be implemented efficiently by transmitting a single message.

Processes are modeled so as not to exhibit faulty behavior, i.e., they correctly follow the protocol until termination. The notion of a faulty process is instead captured by the assumption of faulty message transmissions. For example, a crashed process can be expressed by the loss of every message transmitted by it. The model considers omission transmission failures. A transmission between two processes $p_{i}$ and $p_{j}$ is subject to an omission failure if the message sent by $p_{i}$ is not received by $p_{j}$.

In rounds where omission faults are bounded by $f \leq\left\lceil\frac{n}{2}\right\rceil(n-k)+k-2$ out of the $n^{2}$ transmissions that occur (where $k$ is the number of processes required to decide), the protocol necessarily makes some progress that eventually leads to a decision. Therefore, if enough of these rounds occur, then the protocol ensures termination with probability 1 . Nevertheless, to simplify the correctness proofs we will assume that there is some unknown time after which at most $f$ faulty transmissions occur at each round. The number of faults per round prior to this is unrestricted and can for instance match the total number of transmissions $n^{2}$.

Finally, every process $p_{i} \in \Pi$ has access to a local random bit generator that returns unbiased bits observable only by $p_{i}$, and access to a function $\#_{x}(V)$ that returns the number of occurrences of an element $x$ in a vector $V$.

\section{The Algorithm}

This section presents a $k$-consensus algorithm (Algorithm 1). The algorithm is tolerant to omission faults and relies on each process $p_{i}$ having access to a local coin $^{1}$ mechanism that returns random bits observable only by $p_{i}$ (e.g., $\left.[3,5]\right)$. Safety (i.e., the validity and agreement properties of consensus) is ensured by the algorithm regardless of the number of omission faults that occur per round, while liveness (i.e., the termination property) is ensured if, after some arbitrary number of rounds, the number of omission faults per round does not exceed the threshold $f \leq\left\lceil\frac{n}{2}\right\rceil(n-k)+k-2$.

The internal state of a process $p_{i}$ is comprised by three variables: (1) the phase $\phi_{i} \geq 1,(2)$ the proposal value $v_{i} \in\{0,1\}$, and finally, (3) the decision status status $_{i} \in\{$ decided, undecided $\}$. Each process starts its execution with $\phi_{i}=1$, status $_{i}=$ undecided, while $v_{i}$ is set to the initial proposal value indicated by the input register proposal $_{i}$.

A round of the algorithm is executed as follows. Upon every clock tick (line 5), each process $p_{i}$ broadcasts a message of the form $\left\langle\phi_{i}, v_{i}\right.$, status $\left._{i}\right\rangle$ containing the variables that comprise its internal state, and receives the messages broadcast by all processes (lines 6-7). Some of the messages that a process is supposed to receive may be lost. Any new messages that a process $p_{i}$ receives at every round are accumulated in a vector $V_{i}$ (line 8 ). A message $\langle\phi, v$, status $\rangle$ transmitted by a process $p_{j}$ is considered new if it does not exist in $V_{i}$ any message with phase

\footnotetext{
${ }^{1}$ As opposed to a shared coin that returns bits observable by all processes (e.g., [25,
} $6])$. 


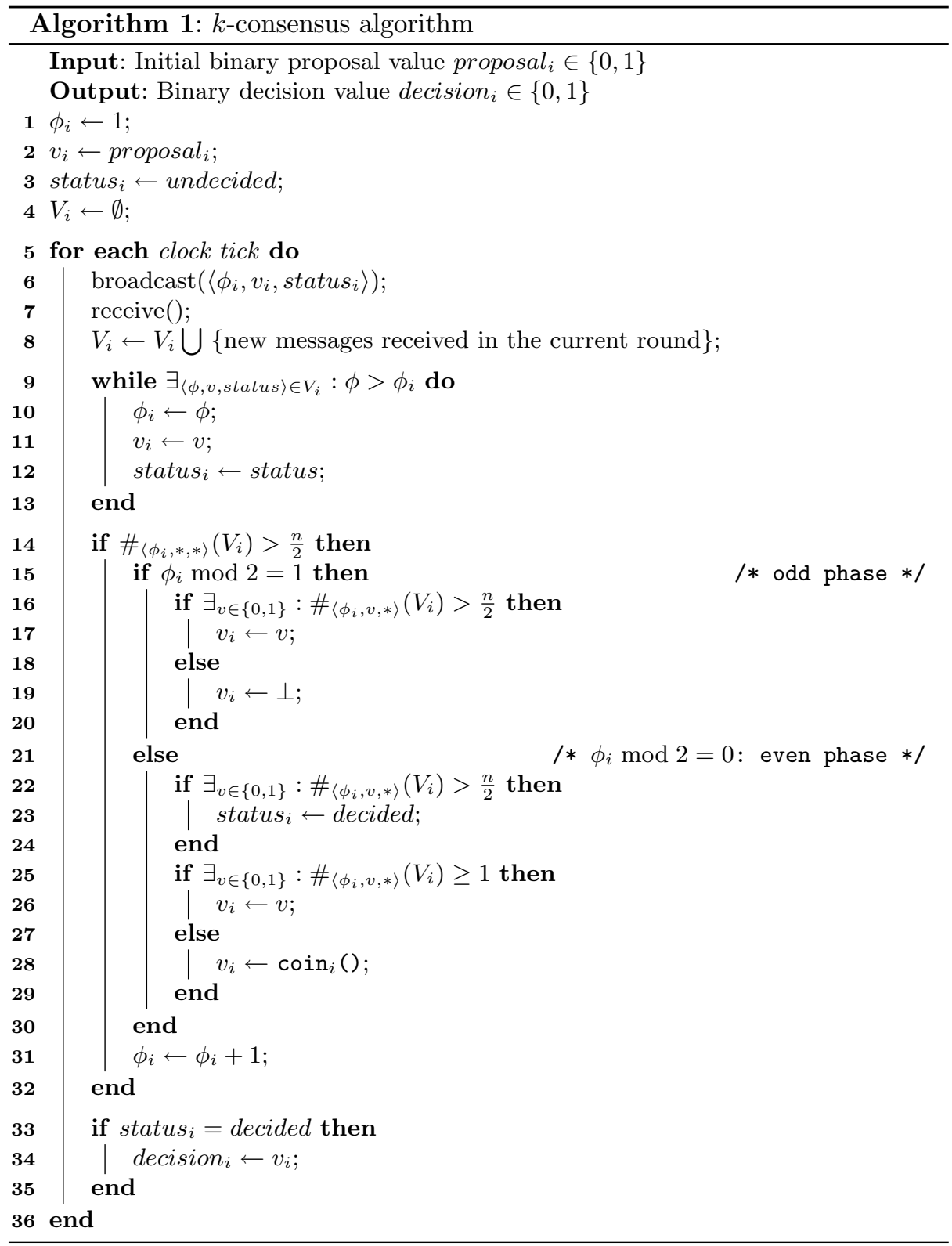

value $\phi$ from $p_{j}$. This implies that it is impossible to accumulate in vector $V_{i}$ more than one message with the same phase value $\phi$ from any single process. Based on its current internal state and the messages accumulated so far in vector $V_{i}$, each process $p_{i}$ performs a state transition (i.e., modifies $\phi, v$ or status). 
Before explaining how a process performs a state transition, it is important to note the distinction between round and phase. The term round pertains to a periodic execution of the protocol activated by a synchronous event, a clock tick in this case. The term phase pertains to a monotonic variable $\phi_{i}$ that is part of the internal state of a process $p_{i}$, and whose value increases as $p_{i}$ accumulates messages of a certain form in vector $V_{i}$. How exactly $\phi_{i}$ is updated is explained below. For now, it is beneficial to retain that for any given round, any two processes $p_{i}$ and $p_{j}$ can have different phase values $\phi_{i} \neq \phi_{j}$.

A process $p_{i}$ performs a state transition when one of two conditions occur:

1. The vector $V_{i}$ holds one message from some process $p_{j}$ whose phase $\phi_{j}$ is higher than the phase $\phi_{i}$ of $p_{i}$.

2. The vector $V_{i}$ holds more than $\frac{n}{2}$ messages whose phase is equal to the phase $\phi_{i}$ of $p_{i}$.

The first case is straightforward (lines 9-13). When the condition is met (line $9)$, the process $p_{i}$ updates its state to match exactly the state of the received message (lines 10-12).

The second case is more complex (lines 14-32). The way a process $p_{i}$ updates its state depends on whether the current number of its phase $\phi_{i}$ is odd (i.e., $\phi_{i} \bmod 2=1$ ) or even (i.e., $\phi_{i} \bmod 2=0$ ). The odd phase essentially guarantees that if two processes set their proposal to a value 1 or 0 , they do it for the same value. The even phase is where a process decides if it learns that a majority of processes have the same proposal value.

If $\phi_{i} \bmod 2=1$ (lines 15-20), then the proposal value $v_{i}$ is updated in the following way: if there are more than $\frac{n}{2}$ messages of the form $\left\langle\phi_{i}, v, *\right\rangle$ in $V_{i}$ with the same value $v$, then $v_{i}$ is set to $v$ (lines 16-17), otherwise it is set to a special value $\perp \notin\{0,1\}$ indicating a lack of preference (lines 18-19).

If $\phi_{i} \bmod 2=0$ (lines 21-30), then the process sets status $s_{i}$ to decided if there are more than $\frac{n}{2}$ messages of the form $\left\langle\phi_{i}, v, *\right\rangle$ in $V_{i}$ with the same value $v \neq \perp$ (lines 22-24). The proposal value $v_{i}$ is updated to $v$ if there is at least one message of the form $\left\langle\phi_{i}, v, *\right\rangle$ in $V_{i}$ with a value $v \neq \perp$, otherwise $v_{i}$ is set to the value of function $\operatorname{coin}()$, which returns a random number 0 or 1 , each with a probability $\frac{1}{2}$ (lines 25-29). Regardless of whether the phase $\phi_{i}$ is odd or even, its value is always incremented by one unit at line 31 .

At the end of each round, a process $p_{i}$ checks if status $s_{i}$ has been set to decided. If so, it decides by setting the output variable decision de $_{i}$ to the current proposal value $v_{i}$ (lines 33-35). Any further accesses to this variable do not alter its value. Hence, they have no impact on the correctness of the algorithm.

In the presented algorithm, processes do not voluntarily stop sending messages. The fact that the system stabilization time is unknown combined with the assumed fault model means that processes have no way of knowing when other processes have decided. This limitation can be easily overcome by having the processes execute for an additional round after deciding, where the broadcast operation is performed through a reliable (and possibly asynchronous) channel. Raynal and Roy showed that it is possible to implement reliable and asynchronous communication on top of an unreliable and synchronous model, and 
vice-versa [26]. One can assume the presence of a reliable channel that is judiciously used in such situations.

\section{Correctness Proof}

In this section we prove the correctness of the algorithm. Up to Theorem 2 we prove validity and agreement properties, which are made on the assumption that the system might be subject to an unbounded number of faults per round. From Lemma 4 and on, we address the termination property and assume the number of faults per round is $f \leq\left\lceil\frac{n}{2}\right\rceil(n-k)+k-2$.

Lemma 1. If every process $p_{i}$ with phase value $\phi_{i}=\phi$ has the same proposal value $v_{i}=v$, then every process $p_{j}$ that sets $\phi_{j}=\phi+1$ also sets $v_{j}=v$.

Proof. The lemma is going to be proved by induction on the number of processes that reach phase $\phi+1$. Basis: Without loss of generality, let $p_{1}$ be the first process that sets $\phi_{1}=\phi+1$. In this case, process $p_{1}$ must have received more than $\frac{n}{2}$ messages of the form $\langle\phi, *, *\rangle$ (Line 14). Since every process $p_{i}$ with $\phi_{i}=\phi$ has the same value $v_{i}=v$, every broadcast message of the form $\langle\phi, *, *\rangle$ carries the same proposal value $v$ (Line 6 ). This implies that the more than $\frac{n}{2}$ messages received by process $p_{1}$ have the form $\langle\phi, *, *\rangle$ with the same value $v$. Therefore, $p_{1}$ must set its proposal value to $v$ (either on Line 17 or 26). Inductive step: Assume that every process $p_{u}$ with $1 \leq u \leq j-1$ has $\phi_{u}=\phi+1$ and $v_{u}=v$, and now we want to demonstrate that when $p_{j}$ sets $\phi_{j}=\phi+1$ it will also set $v_{j}=v$. In order for process $p_{j}$ to set $\phi_{j}=\phi+1$ it must have in vector $V_{j}(1)$ more than $\frac{n}{2}$ messages of the form $\langle\phi, *, *\rangle$ (Line 14) or (2) at least a message of the form $\langle\phi+1, *, *\rangle$ (Line 9). Condition (1) corresponds to the basis case, and therefore it has already been shown that $p_{j}$ sets $v_{j}=v$. Condition (2) also results in the same outcome, since by hypothesis message $\langle\phi+1, *, *\rangle$ must have been transmitted by one of the $p_{u}$ processes, and therefore $p_{j}$ also sets $\phi_{j}=\phi+1$ and $v_{j}=v$ (Lines 10-11).

Lemma 2. Let $\phi$ be some odd phase (i.e., $\phi \bmod 2=1$ ). If every process with phase value $\phi$ has the same proposal value $v$, then every process that sets its phase to any value $\phi^{\prime}>\phi+1$ decides $v$.

Proof. Since every process with odd phase value $\phi$ has the same proposal value $v$, by Lemma 1, every process that reaches even phase $\phi+1$ also has proposal value $v$ (either on Lines $10-11$ or Lines 17 and 31 ). Let $p_{i}$ be the first process to set phase value $\phi_{i}=\phi+2$. Since there is no other process $p_{j}$ with phase value $\phi_{j}>\phi+1$, the only way for $p_{i}$ to go from phase $\phi+1$ to $\phi+2$ is to receive more than $\frac{n}{2}$ messages of the form $\langle\phi+1, *, *\rangle$ (Line 14). Since $\phi+1$ is even and all these messages carry the same proposal value $v$, this implies that $p_{i}$ sets status $_{i}=$ decided, $v_{i}=v$ and $\phi_{i}=\phi+2$ (Lines 23, 26, 31). Consequently, process $p_{i}$ can now decide $v$ (Line 34 ).

The next process that sets its phase value to $\phi+2$ also decides $v$ because it either accumulates more than $\frac{n}{2}$ messages with phase value $\phi+1$ and same 
proposal value $v$ (Lines 23, 26, 31 and 34), or receives a message from $p_{i}$ of the form $\langle\phi+2, v$, decided $\rangle$ (Lines 10-12 and 34). This reasoning can be applied recursively to any other process that sets its phase value to $\phi+2$. It follows that any process that sets its phase value to $\phi^{\prime} \geq \phi+2$ must either had been at phase $\phi+2$, and hence decided, or it must have received some message from a process that went through phase $\phi+2$, and thus also deciding. Therefore, every process that sets its phase to any value $\phi^{\prime}>\phi+1$ decides $v$.

Theorem 1. If all processes propose the same value $v$, then every process that decides, decides $v$.

Proof. If every process has the same initial proposal value $v$, then they all start in odd phase 1 and set proposal value to $v$ (Lines 1-2). Therefore, by Lemma 1 , every process $p_{j}$ that sets phase $\phi_{j}=2$ also has proposal value $v_{j}=v$. Moreover, by Lemma 2 , every process $p_{i}$ that sets its phase to $\phi_{i}>2$, decides $v$.

Lemma 3. In some even phase $\phi$, there are no two process $p_{i}$ and $p_{j}$ that receive messages of the form $\langle\phi, 0, *\rangle$ and $\langle\phi, 1, *\rangle$, respectively.

Proof. Suppose otherwise. Then $p_{i}$ and $p_{j}$ are two processes with phase value $\phi$ that, respectively, receive a message $\langle\phi, 0, *\rangle$ from $p_{u}$ and a message $\langle\phi, 1, *\rangle$ from $p_{w}$. This implies that process $p_{u}$ sets $v_{u}=0$ either because on odd phase $\phi-1$ it accumulated more than $\frac{n}{2}$ messages of the form $\langle\phi-1,0, *\rangle$ (Lines 16-17, 31), or because it received a message $\langle\phi, 0, *\rangle$ (Lines 10-11) from a process that had accumulated that majority of $\langle\phi-1,0, *\rangle$ messages. Using a similar reasoning, in order for process $p_{w}$ to set $v_{w}=1$, some process must have received on odd phase $\phi-1$ more than $\frac{n}{2}$ messages of the form $\langle\phi-1,1, *\rangle$. But this is a contradiction because only one of the proposal values 0 and 1 can be in a majority of the messages broadcast for any particular phase number.

Theorem 2. No two processes decide differently.

Proof. Let $p_{i}$ be the first process to decide, and do so when phase $\phi_{i}=\phi$ (Line $34)$. Without loss of generality, let the decision value be 1 . Then, vector $V_{i}$ must contain more than $\frac{n}{2}$ messages of the form $\langle\phi-1,1$, undecided $\rangle$, and $\phi-1$ must be even (to allow the execution of Lines 23, 26, and 31). By Lemma 3, no other process $p_{j}$ can receive a message of the form $\langle\phi-1,0, *\rangle$. Therefore, every other process $p_{j}$ with phase $\phi_{j}=\phi$ has proposal value $v_{j}=1$ either because it accumulates more than $\frac{n}{2}$ messages with at least one being of the form $\langle\phi-1,1, *\rangle$ (Line 26$)$, or because it receives a message $\langle\phi, 1, *\rangle$ (Line 11) transmitted by process $p_{i}$ (or another process that sets its proposal value to 1 ). Additionally, since all processes with phase $\phi$ have proposal value 1 , then by Lemmas 1 and 2, every process that decides in phase $\phi^{\prime}>\phi$ will do it for value 1.

The remainder of the proof serves to prove the termination property of consensus (Theorem 3) and is made on the assumption that the message scheduling falls under the control of an adversary that can cause no more than $f$ faults per round for $f \leq\left\lceil\frac{n}{2}\right\rceil(n-k)+k-2$. 
Lemma 4. If some process $p_{i}$ has some phase value $\phi_{i}>1$, then there is a set of processes $S$ such that $\forall_{p_{j} \in S}: \phi_{j} \geq \phi_{i}-1$ and $|S|>\frac{n}{2}$.

Proof. Given a phase number $\phi>1$, then there must be some process $p_{i}$ that is the first to set its phase to $\phi_{i}=\phi$. In order to do this, $p_{i}$ must have more than $\frac{n}{2}$ messages of the form $\langle\phi-1, *, *\rangle$ in vector $V_{i}$ (Line 14). It follows that there are more than $\frac{n}{2}$ processes that were at some point in time in phase $\phi-1$.

Lemma 5. If some process $p_{i}$ has phase value $\phi_{i}=\phi$, then eventually there is a set of processes $S$ such that $\forall_{p_{j} \in S}: \phi_{j} \geq \phi-1$ and $|S| \geq k$.

Proof. Suppose otherwise. By Lemma 4 , if some process $p_{i}$ has $\phi_{i}=\phi>1$, then there is a set of processes $S$ such that $\forall_{p_{j} \in S}: \phi_{j} \geq \phi-1$ and $|S|>\frac{n}{2}$. Let $R^{+}=S$ where $\frac{n}{2}<\left|R^{+}\right|<k$, and $R^{-}$be the set of remaining processes, i.e., $\forall_{p_{u} \in R^{-}}: \phi_{u}<\phi-1$ where $n-k<\left|R^{-}\right|<\frac{n}{2}$.

By assumption, the adversary can create at most $f=f_{1}+f_{2}$ message omissions per round, where $f_{1}=\left\lceil\frac{n}{2}\right\rceil(n-k)$ and $f_{2}=k-2$. In order to prevent processes in $R^{-}$from reaching $\phi_{u} \geq \phi-1$, the adversary must omit every message from processes of $R^{+}$to $R^{-}$(due to Lines 9-13). This implies the elimination of more than $\frac{n}{2}$ messages in more than $n-k$ processes because $\left|R^{+}\right|>\frac{n}{2}$ and $\left|R^{-}\right|>n-k$. It is clear that after consuming $f_{1}$ faults, there are at most $n-k$ processes in $R^{-}$that do not receive any message from $R^{+}$.

Since by definition $\left|R^{-}\right|-(n-k)=k-\left|R^{+}\right|>0$, there must be $k-\left|R^{+}\right|$ processes in $R^{-}$that could still receive messages from every process in $R^{+}$. Let $R_{*}^{-}$denote the set of processes in this situation. To prevent every process $p_{u}$ in $R_{*}^{-}$from reaching $\phi_{u} \geq \phi-1$, the adversary must create $\left|R^{+}\right|\left|R_{*}^{-}\right|$omissions, where $\left|R^{+}\right|+\left|R_{*}^{-}\right|=k$. However, the adversary only has $f_{2}=k-2=\left|R^{+}\right|+$ $\left|R_{*}^{-}\right|-2$ faults available. This creates a contradiction because $\left|R^{+}\right|\left|R_{*}^{-}\right|>\left|R^{+}\right|+$ $\left|R_{*}^{-}\right|-2$, for all $\left|R^{+}\right| \geq 1$ and $\left|R_{*}^{-}\right| \geq 1$. This implies that some process in $\left|R^{-}\right|$ always increases its phase value when $\frac{n}{2}<\left|R^{+}\right|<k$.

Lemma 6. Let $R^{+}$be the set of processes such that $\forall_{p_{i} \in R^{+}}: \phi_{i} \geq \phi$, with $\left|R^{+}\right|=k+\alpha$ and $0 \leq \alpha \leq n-k$. Let $\alpha$ or more processes in $R^{+}$have phase $\phi$ and the remaining processes of $R^{+}$have phase $\phi+1$. Let $R^{-}$be the set of process such that $\forall_{p_{j} \in R^{-}}: \phi_{j}<\phi$, with $\left|R^{-}\right|=n-k-\alpha$. Whenever a round has such configuration, some process increases its phase value.

Proof. Suppose otherwise. Then, under the Lemma conditions, there must be a message schedule where at some round no process increases its phase value.

In order to prevent every process in $R^{-}$from increasing its phase value, the adversary must omit every message from $R^{+}$to $R^{-}$(due to Lines 9-13). This requires that $\left|R^{+}\right|\left|R^{-}\right|$faults must be spent. Since $\left|R^{+}\right|\left|R^{-}\right|=(k+\alpha)(n-k-\alpha)$ and the total number of omissions per round is $f=\left\lceil\frac{n}{2}\right\rceil(n-k)+k-2$, then the adversary is left with no more than $f-\left|R^{+}\right|\left|R^{-}\right| \leq\left(\alpha+\left\lceil\frac{n}{2}\right\rceil+k-n\right) \alpha+k-2$ faults.

In order to block each of the $\alpha$ processes in $R^{+}$with phase $\phi$, the adversary must omit all messages from processes in $R^{+}$with phase $\phi+1$ (Line 9) and it 
must prevent the reception of more than $\frac{n}{2}$ messages of the form $\langle\phi, *, *\rangle$ also from processes in $R^{+}$(Line 14). This implies that each of the $\alpha$ processes with phase $\phi$ can receive the $n-k-\alpha$ messages from processes in $R^{-}$and at most $\left\lfloor\frac{n}{2}\right\rfloor$ messages from processes in $R^{+}$. Therefore, the adversary must create at least $\left[n-\left(\left\lfloor\frac{n}{2}\right\rfloor+n-k-\alpha\right)\right] \alpha$ faults to stop the progression of the $\alpha$ processes. Since $\left[n-\left(\left\lfloor\frac{n}{2}\right\rfloor+n-k-\alpha\right)\right] \alpha=\left(\alpha+\left\lceil\frac{n}{2}\right\rceil+k-n\right) \alpha$, the adversary is left with no more than $k-2$ faults.

For the remaining $k$ processes in $R^{+}$, there are two possible cases:

1. First consider the two extreme situations, where all $k$ processes either have phase value $\phi$ or $\phi+1$. Since the adversary only has $k-2$ faults left, some process has to receive more than $\frac{n}{2}$ messages with the same phase $\phi$ or $\phi+1$. Therefore, some process increases its phase value (Line 14).

2. Second consider that some of the $k$ processes have phase value $\phi+1$ and the others have phase value $\phi$. Let $H$ be the set of processes with $\phi+1$ and $L$ the set of processes with $\phi$, such that $|H|+|L|=k$. To block the processes in $L$, the adversary has to omit $|H||L|$ messages (due to Line 9). Since the adversary only has $k-2=|H|+|L|-2$ faults left, it cannot prevent some process from increasing its phase because $|H||L|>|H|+|L|-2$ for all $|H| \geq 1$ and $|L| \geq 1$.

Lemma 7. Let $\phi_{\text {init }}=1$ be the initial phase value for all processes. Some process $p_{i}$ eventually sets $\phi_{i}>\phi_{\text {init }}$.

Proof. If every process has the same phase value $\phi_{\text {init }}$, then according to the conditions of Lemma 6 , this is equivalent of having every process in set $R^{+}$with phase $\phi_{\text {init }}$, such that $\left|R^{+}\right|=n$. Therefore, by Lemma 6 , some process has to increase its phase value and set $\phi_{i}>\phi_{\text {init }}$.

Lemma 8. If some process has phase value $\phi$, then eventually some process must have phase value $\phi+1$.

Proof. If some process has phase value $\phi$, then by Lemma 5, eventually there is a set $R^{+}$of $k$ or more processes such that $\forall_{p_{i} \in R^{+}}: \phi_{i} \geq \phi-1$. This implies that the system must reach a configuration where there are two sets of processes $R^{+}$ and $R^{-}$according to the conditions of Lemma 6 . When this happens, by the same Lemma, some process will increase its phase. This process can be in one of three possible cases: (1) a process of $R^{-} ;(2)$ a process with phase number $\phi-1$ of $R^{+}$; or (3) a process with phase number $\phi$ of $R^{+}$. The system configuration resulting from cases (1) and (2) falls under the conditions of Lemma 6 , and therefore more processes will continue to increase their phase. Consequently, in the most extreme scenario, the system will evolve to a configuration where all process are in phase number $\phi$, and case (3) will necessarily have to occur, and some process $p_{i}$ will set its phase number to $\phi_{i}=\phi+1$.

Theorem 3. At least $k$ processes eventually decide with probability 1. 
Proof. The proof is organized in two parts. First, we show that as messages are received, processes make progress on the protocol execution and continue to increase their phase number. Second, we demonstrate that due to this progression, eventually the system will reach to a configuration where at least $k$ processes decide with probability 1.

First part: By Lemma 7, some process $p_{i}$ eventually increases its phase number from the initial phase number, i.e., $\phi_{i}=\phi>\phi_{\text {init }}$. Then, by Lemma 8 , some process will eventually set its phase number to $\phi+1$. Moreover, by Lemma 5 , $k$ or more processes set their phase value to at least $\phi$. Since these Lemmas can be applied repeatedly, this ensures that at least a set of $k$ processes continue to increase their phase numbers.

Second part: By Lemma 3, no two processes with the same even phase value $\phi$ can receive messages $\langle\phi, 0, *\rangle$ and $\langle\phi, 1, *\rangle$. Therefore, any process $p_{i}$ that enters the if condition of Line 14, and sets $\phi_{i}=\phi+1$ (Line 31), must set its proposal value $v_{i}$ either to a common value $v$ (Line 26) or to a random value 1 or 0 (Lines 28 ). Let $S$ be the processes that eventually reach phase value $\phi+1$, with $|S| \geq k$ due to the above discussion. Then, at least $k$ processes in $S$ will set their proposal values to the same $v$ with probability $p=2^{-k}$. Therefore, the probability that $k$ processes do not set the same proposal value $v$ is $(1-p)$.

As the protocol progresses, and the phase number of processes increases, the probability of not existing a phase where $k$ processes propose the same value $v$ is $\lim _{\phi \rightarrow \infty}(1-p)^{\phi}=0$. Thus, eventually there will be a phase $\phi_{t}$ where $k$ processes have the same proposal value $v$ with probability 1 . According to Lemma 2, every process that sets its phase value to $\phi>\phi_{t}$ decides $v$. Consequently, at least $k$ processes decide.

\section{$7 \quad$ Performance}

The algorithm guarantees the termination property of consensus in a probabilistic fashion. Since the execution of the algorithm may need to extend for any number of rounds and any process may reach an arbitrarily high phase, eventually there will be a phase where all processes flip the same coin value $v$ and decide (Theorem 3). The number of expected rounds for this to happen is $O\left(2^{n}\right)$ after the system stabilizes in at most $f$ faults per round. Note that this is the most extreme possible scenario. In fact, the presence of an adversary that enforces a worst-case scheduling is very unlikely to happen in practice [19, 20]. A simple inspection of the protocol suffices to observe that the algorithm is fastlearning, i.e., it decides within two communication rounds in runs with no faults or with certain fault patterns. This is true even if processes have different initial proposal values. As long as the fault distribution is benign enough, $k$ processes will see the majority of one value during the first phase, propose the same value for the second phase and decide. 


\section{Conclusions}

Despite its usefulness to represent wireless ad-hoc communication environments, research on the communication failure model has been limited. This is related to an associated impossibility result, which states that no agreement is possible in a synchronous system if at every communication round more than $n-2$ messages can be lost $[27,28]$. This paper presents a $k$-consensus algorithm tolerant to transmission omission faults, the first to circumvent the Santoro-Widmayer impossibility result using randomization. In a system with $n$ processes, our algorithm makes consensus possible among $k>\frac{n}{2}$ processes. It maintains safety despite an unrestricted number of faults and ensures liveness if the number of omission faults does not exceed $\left\lceil\frac{n}{2}\right\rceil(n-k)+k-2$. Furthermore, the algorithm can be fast learning in the sense that it terminates in two communication steps under favorable conditions.

\section{References}

1. M. Aguilera, W. Chen, and S. Toueg. Failure detection and consensus in the crash-recovery model. Distributed Computing, 13(2):99-125, 2000.

2. E. A. Akkoyunlu, K. Ekanadham, and R. V. Huber. Some constraints and tradeoffs in the design of network communications. In Proceedings of the 5th ACM Symposium on Operating Systems Principles, pages 67-74, 1975.

3. M. Ben-Or. Another advantage of free choice: Completely asynchronous agreement protocols. In Proceedings of the 2nd ACM Symposium on Principles of Distributed Computing, pages 27-30, 1983.

4. M. Biely, J. Widder, B. Charron-Bost, A. Gaillard, M. Hutle, and A. Schiper. Tolerating corrupted communication. In Proceedings of the 26th ACM Symposium on Principles of Distributed Computing, pages 244-253, 2007.

5. G. Bracha. An asynchronous $\lfloor(n-1) / 3\rfloor$-resilient consensus protocol. In Proceedings of the 3rd ACM Symposium on Principles of Distributed Computing, pages 154-162, 1984.

6. C. Cachin, K. Kursawe, and V. Shoup. Random oracles in Constantinople: Practical asynchronous Byzantine agreement using cryptography. Journal of Cryptology, 18(3):219-246, 2005.

7. T. Chandra and S. Toueg. Unreliable failure detectors for reliable distributed systems. Journal of the ACM, 43(2):225-267, 1996.

8. B. Charron-Bost and A. Schiper. The heard-of model: Computing in distributed systems with benign failures. Technical Report LSR-REPORT-2007-001, EPFL, 2007.

9. G. Chockler, M. Demirbas, S. Gilbert, N. Lynch, C. Newport, and T. Nolte. Consensus and collision detectors in radio networks. Distributed Computing, 21(1):55$84,2008$.

10. D. Dolev, C. Dwork, and L. Stockmeyer. On the minimal synchronism needed for distributed consensus. Journal of the ACM, 34(1):77-97, 1987.

11. D. Dolev, R. Friedman, I. Keidar, and D. Malkhi. Failure detectors in omission failure environments. In Proceedings of the 16th ACM Symposium on Principles of Distributed Computing, pages 286-295, 1997. 
12. M. J. Fischer. The consensus problem in unreliable distributed systems (A brief survey). In M. Karpinsky, editor, Foundations of Computing Theory, volume 158 of Lecture Notes in Computer Science, pages 127-140. Springer, 1983.

13. M. J. Fischer, N. A. Lynch, and M. S. Paterson. Impossibility of distributed consensus with one faulty process. Journal of the ACM, 32(2):374-382, 1985.

14. J. Gray. Notes on data base operating systems. In R. Bayer, R. M. Graham, and G. Seegmüller, editors, Operating Systems: An Advanced Course, volume 60 of Lecture Notes in Computer Science. Springer-Verlag, 1978.

15. M. Hurfin, A. Mostefaoui, and M. Raynal. Consensus in asynchronous systems where processes can crash and recover. In Proceedings of the the 17th IEEE Symposium on Reliable Distributed Systems, pages 280-286, 1998.

16. L. Lamport. Lower bounds for asynchronous consensus. Distributed Computing, 19(2):104-125, 2006.

17. L. Lamport, R. Shostak, and M. Pease. The Byzantine generals problem. ACM Transactions on Programming Languages and Systems, 4(3):382-401, 1982.

18. N. A. Lynch. Distributed Algorithms. Morgan Kaufmann, 1997.

19. H. Moniz, N. F. Neves, M. Correia, and P. Veríssimo. Experimental comparison of local and shared coin randomized consensus protocols. In Proceedings of the 25th IEEE Symposium on Reliable Distributed Systems, pages 235-244, 2006.

20. H. Moniz, N. F. Neves, M. Correia, and P. Veríssimo. RITAS: Services for randomized intrusion tolerance. IEEE Transactions on Dependable and Secure Computing, to appear, 2009.

21. N. F. Neves, M. Correia, and P. Veríssimo. Solving vector consensus with a wormhole. IEEE Transactions on Parallel and Distributed Systems, 16(12):1120-1131, 2005.

22. R. Oliveira, R. Guerraoui, and A. Schiper. Consensus in the crash-recover model. Technical Report 97-239, EPFL, 1997.

23. M. Pease, R. Shostak, and L. Lamport. Reaching agreement in the presence of faults. Journal of the ACM, 27(2):228-234, 1980.

24. K. J. Perry and S. Toueg. Distributed agreement in the presence of processor and communication faults. IEEE Transactions on Software Engineering, 12(3):477-482, 1986.

25. M. O. Rabin. Randomized Byzantine generals. In Proceedings of the 24th Annual IEEE Symposium on Foundations of Computer Science, pages 403-409, 1983.

26. M. Raynal and M. Roy. A note on a simple equivalence between round-based synchronous and asynchronous models. In Proceedings of the 11th IEEE Pacific Rim International Symposium on Dependable Computing, pages 387-392, 2005.

27. N. Santoro and P. Widmayer. Time is not a healer. In Proceedings of the 6th Symposium on Theoretical Aspects of Computer Science, pages 304-313, 1989.

28. N. Santoro and P. Widmayer. Agreement in synchronous networks with ubiquitous faults. Theoretical Computer Science, 384(2-3):232-249, 2007.

29. U. Schmid, B. Weiss, and I. Keidar. Impossibility results and lower bounds for consensus under link failures. SIAM Journal on Computing, 38(5):1912-1951, 2009.

30. G. Varghese and N. A. Lynch. A tradeoff between safety and liveness for randomized coordinated attack. Information and Computation, 128(1):57-71, 1996. 